\title{
Spatial memory in the Morris water maze and activation of cyclic AMP response element-binding (CREB) protein within the mouse hippocampus
}

\author{
Yves Porte, Marie Christine Buhot, and Nicole E. Mons ${ }^{1}$ \\ CNRS UMR 5228, Centre de Neurosciences Intégratives et Cognitives, 33405 Talence, France
}

\begin{abstract}
We investigated the spatio-temporal dynamics of learning-induced cAMP response element-binding protein activation/phosphorylation (pCREB) in mice trained in a spatial reference memory task in the water maze. Using immunohistochemistry, we examined pCREB immunoreactivity (pCREB-ir) in hippocampal CAl and CA3 and related brain structures. During the course of spatial learning over Days 1-9, pCREB-ir progressively increased in hippocampal neurons whereas its level in the dorsal striatum decreased. No significant changes were observed in the prelimbic cortex and lateral amygdala. Mice killed at various time points after the last training session demonstrated two waves of pCREB-ir in CAI and an early transient CREB phosphorylation in area CA3, lateral amygdala, and prelimbic cortex. We show that CREB phosphorylation and downstream gene Zif268 activation remained sustained in $\mathrm{CA} 1$ and $\mathrm{CA} 3$ for at least $24 \mathrm{~h}$ after extended training (Days 8-9) but not during early training (Day 3). The present results indicate that the strong CAl CREB phosphorylation observed immediately after training was not related strictly to learning or to memory. In contrast, at 15 min after training, the changes in CAI CREB phosphorylation state were specifically related to individual learning capability. We suggest that hippocampal-learning specificity of CREB is reflected best by duration, rather than magnitude, of CREB phosphorylation.
\end{abstract}

Memory can be divided into at least two distinct forms according to its temporal and biochemical properties: short term memory (STM), which lasts no longer than a few hours, and long-term memory (LTM), which lasts from several hours to days or even longer (McGaugh 1966; Davis and Squire 1984; Matthies 1989; Bozon et al. 2003a,b). Studies in rodents have provided evidence that STM is a labile state that depends on activation and/or posttranslational modifications of preexisting molecules whereas consolidation of LTM depends on a crucial phase of gene expression and de novo protein synthesis to transform newly learned information into a permanent and stable state (Davis and Squire 1984; Dudai 1996; McGaugh 2000). Numerous studies have shown that consolidation of many types of LTM in rodents requires phosphorylation/activation of the transcription factor CREB (cAMP response element-binding protein) on Ser ${ }^{133}$ by cAMP- or $\mathrm{Ca}^{2+}$-dependent protein kinase (Bernabeu et al. 1997; Izquierdo and Medina 1997; Impey et al. 1998; Taubenfeld et al. 1999; Cammarota et al. 2000, 2005; Vianna et al. 2000; Izquierdo et al. 2001; Stanciu et al. 2001; Kida et al. 2002; Desmedt et al. 2003; Zhang et al. 2003; Countryman et al. 2005; Trifilieff et al. 2006; Brightwell et al. 2007). We and others recently showed that spatial memory formation was associated with increased CREB phosphorylation within the hippocampus (Mizuno et al. 2002; Colombo et al. 2003; Goldbart et al. 2003; Martel et al. 2006; Moncada and Viola 2006; Porte et al.2008). Indeed, a role for CREB in hippocampus-dependent learning was strongly supported by oligonucleotide antisense and transgenic mice studies that showed that a selective suppression of CREB function in hippocampal neurons disrupted consolidation of spatial LTM without affecting task acquisition or STM in a Morris water maze task (Guzowski and McGaugh 1997; Zhang et al. 2003; Florian et al. 2006; but see Balschun et al. 2003).

Although there have been studies proposing that CREB

'Corresponding author.

E-mail n.mons@cnic.u-bordeaux1.fr; fax 33-05-540008743.

Article is online at http://www.learnmem.org/cgi/doi/10.1101//m.1094208. phosphorylation state in the hippocampus may serve as a molecular marker of memory processing for spatial learning (Mizuno et al. 2002; Moncada and Viola 2006), there is little information concerning the spatio-temporal dynamics of CREB phosphorylation within the hippocampus and related structures. We recently provided evidence that the learning-dependent activation of CREB in the hippocampus varies according to the demands relative to the task in which mice were engaged (Trifilieff et al. 2006). Specifically, we found that hippocampal-dependent and -independent behavioral tasks, i.e., contextual and cued conditioning tasks, resulted in distinct (biphasic vs. monophasic, respectively) patterns of hippocampal CREB activation. This suggested that CREB activation may have a key role in delineating learning-dependent circuits as a function of the type of memory processes engaged. The present experiment is aimed at extending these findings by examining the spatial and temporal dynamic of CREB phosphorylation in the hippocampus and related brain structures following acquisition of a spatial reference memory task using the water maze paradigm.

\section{Results}

\section{Behavioral results}

The Ref-4T training protocol leads to a good memory of the platform location

During the spatial reference memory task in the water maze, mice from the Ref-4T group were subjected to one daily session over $9 \mathrm{~d}$ (Fig. 1). On a given day, mice received four acquisition trials during which the hidden platform (PF) was located in a fixed position, i.e., the center of the southwest quadrant (target quadrant).

Figure 2A (black circles) shows performance of mice subjected to the Ref-4T protocol from Day 1 through Day 9. Mice progressively learned the task, as indicated by decreasing latencies from Day 1 to Day 6. This was confirmed by analyses of 


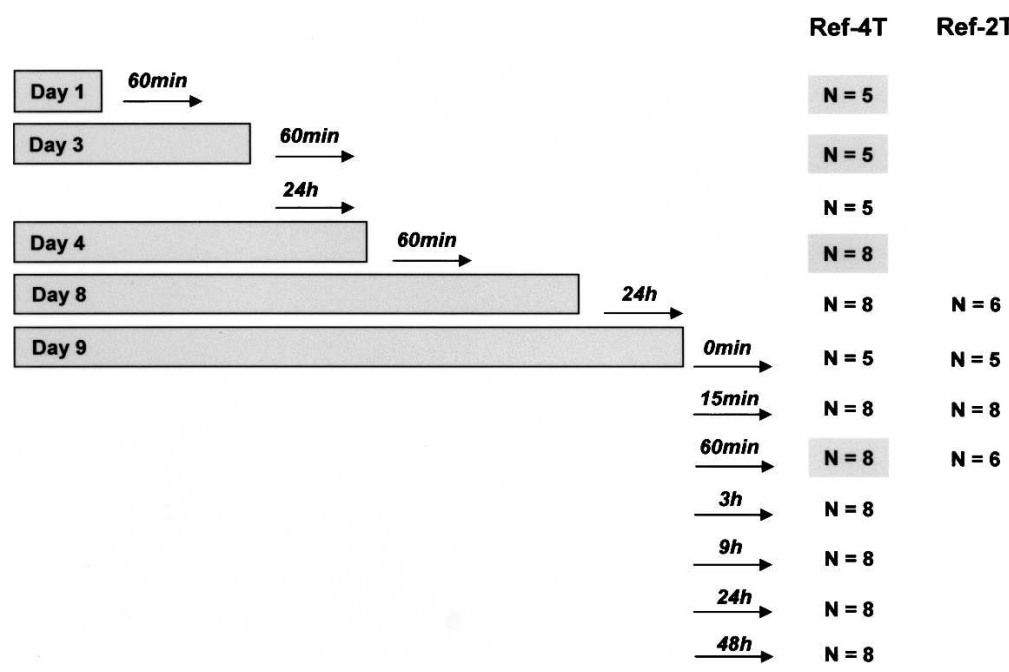

Figure 1. Schematic representation of the experimental procedure used in this study. Mice were submitted to either four (Ref-4T group) or two (Ref-2T group) daily trials in the water maze reference memory task over nine consecutive days. $N$ indicates the number of subjects for each group. Black arrows represent post-training sacrifice time points for $\mathrm{PCREB}$ immunohistochemical analyses. To test whether acquisition of the Ref-4T learning task triggered regional-specific changes in CREB phosphorylation, levels of phosphorylated CREB immunoreactivity (pCREB-ir) were examined 60 min after training on Days 1, 3, 4, and 9. The differential patterns of CREB phosphorylation in hippocampal CA1 and CA3 areas following spatial Ref-4T training were determined at short $(0,15,60 \mathrm{~min})$ and long (3, 9, 24, $48 \mathrm{~h}$ ) time intervals after final probe test on Day 9. Persistence of CREB phosphorylation and Zif268 up-regulation in the hippocampus were studied in Ref-4T animals sacrificed $24 \mathrm{~h}$ after Trial 4 on Days 3 and 8 . Finally, changes in PCREB-ir were examined in four Ref-2T groups, in which memory was not yet consolidated, sacrificed $24 \mathrm{~h}$ after Day 8 or at 0,15 , and 60 min after probe test on Day 9 .

variance performed on data from the whole training period (Day 1 to Day 9), yielding the main effect of Day $\left(F_{(8,416)}=46.669\right.$; $P<0.0001)$. When analyzing performance from Days 1 to 6 , statistical analyses showed significant effects of Day $\left(F_{(5,260)}=33.309 ; P<0.0001\right)$, Trial $\left(F_{(3,156)}=2.997 ; P=0.0325\right)$ but no Day $\times$ Trial interaction $(F<1 ; P>0.5)$. This was mainly due to a strong improvement in latencies from Trial 1 to Trial 4 each day preceding the asymptotic level of performance reached on Day 7 (data not shown).

Memory for the PF location was assessed with a probe trial, which took place immediately after the last trial on Day 9. Animals spent much more time in their target quadrant than in the three other ones (Fig. 2B). ANOVA showed a significant effect of Quadrant $\left(F_{(3,156)}=55.496 ; P<0.0001\right)$ and post-hoc analyses confirmed that the percentage of time spent in the target quadrant was statistically higher than in the three other ones (all $P$ s $<0.0001)$. $t$-Test comparison of the percentage of time spent in the target quadrant further revealed that this bias toward the target quadrant was highly significantly different from chance level $\left(t_{(52)}=10.541 ; P<0.0001\right)$. Moreover, when considering the large PF (LPF), a more restricted area centered on the PF location (see Materials and Methods), analyses of variance showed that Ref-4T mice spent much more time in the target LPF zone than in other equivalent zones of the pool, as revealed by a significant effect of the Zone $\left(F_{(1,52)}=160.631 ; P<0.0001\right.$; Fig. 2C). $t$-Test comparison confirmed that mice from the Ref-4T group spent significantly more time in the target LPF zone than it would be expected by chance $\left(t_{(52)}=11.121 ; P<0.0001\right)$. Taken together, these results indicate that Ref- $4 \mathrm{~T}$ mice displayed a great spatial selectivity toward the target zone.

\section{Behavioral assessment of Ref-2T animals}

An additional experiment was conducted on a group of mice subjected to two daily trials (Ref-2T) throughout Days 1 to 9 . Figure 2A (gray circles) shows the performance of this group over was due to the fact that, unlike the Ref-4T group, mice in the Ref-2T group did not spend more time in the target quadrant than in the three other ones $(F<0.5 ; P>0.5 \mathrm{~ns})$ and relative to chance level $\left(t_{(18)}=0.529 ; P>0.6\right)$. Indeed, post-hoc analyses confirmed that the Ref- $4 \mathrm{~T}$ mice spent a significantly higher percentage of time searching in the target quadrant than Ref-2T animals $(P<0.0001)$. Taken together, our behavioral data indicate that, relative to Ref- $4 \mathrm{~T}$ animals, mice trained in the Ref-2T procedure exhibited a lower encoding of spatial information and performed significantly worse during the retention test.

\section{Immunohistochemical results}

\section{Acquisition of the spatial reference memory task is associated with region-specific changes in phosphorylated CREB}

We and others have previously reported that acquisition of spatial learning was associated with a progressive increase in CREB phosphorylation in the hippocampus without changes in total CREB protein (Mizuno et al. 2002; Colombo et al. 2003; Porte et al. 2008). We first used immunohistochemistry to investigate whether acquisition of the Ref-4T task induced learning-specific, time-dependent changes in the number of pCREB immunoreactive (pCREB-ir) nuclei in the dorsal hippocampus (both CA1-CA3 areas). Figure 3 illustrates the regional changes in pCREB in mice sacrificed 60 min after training on Days 1, 3, 4, and 9. Hippocampal pCREB-ir did not change on Day 1 relative to Naïve controls but, afterward, acquisition of the Ref-4T task was associated with progressively increasing pCREB-ir levels (Fig. 3A). ANOVA of these data revealed a significant Day effect $\left(F_{(3,22)}=8.34\right.$; $P<0.001)$ and post-hoc analyses indicated that acquisition of the spatial task triggered a significant increase in pCREB-ir levels from Day 3 to Day 9 (vs. Naïve: $P \mathrm{~s}<0.01$; Day 3 vs. Day 1: $P<0.05$, and Day 4 or 9 vs. Day 1 : both $P$ s $<0.01$ ). Conversely, the Ref-4T task induced an opposite pattern of pCREB-ir in the striatum (ST; Fig. 3B). Significant increases in ST pCREB-ir oc- 
A

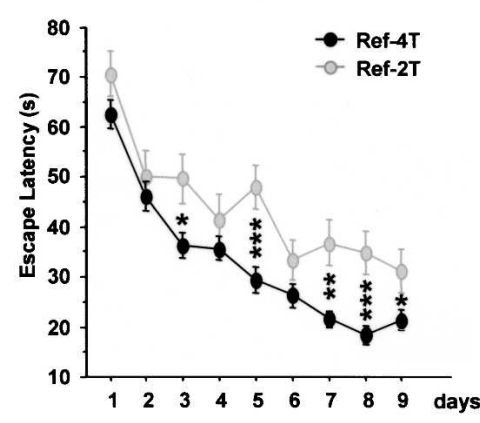

B

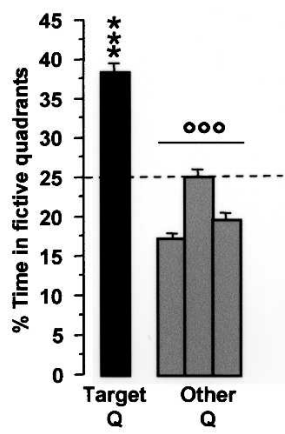

C

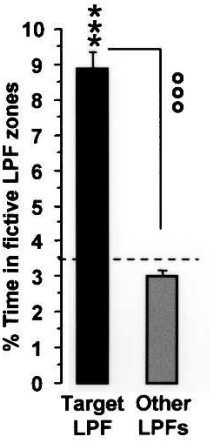

D

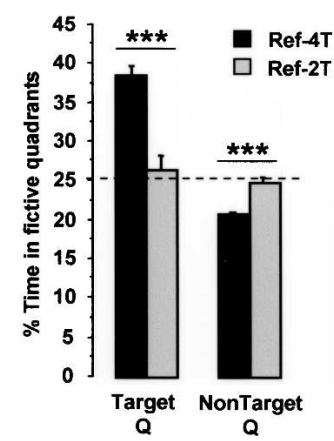

Figure 2. Acquisition and retention of the spatial reference memory task. $(A)$ Acquisition of the reference memory task as expressed by the mean ( \pm SEM) escape latency (in seconds) over nine training sessions (four and two trials/day). Animals performing only two trials per session (Ref-2T group; gray circles) displayed a delayed acquisition compared to animals performing four trials per session (Ref-4T group; black circles), as shown by higher escape latencies from Days 3 to 9 . Memory for the PF location was assessed during the final probe trial (90 s, no PF) on Day 9 . Percentages of time ( \pm SEM) spent in the target quadrant relative to the three other quadrants $(B)$ and in the target LPF area relative to other equivalent zones in the pool ( $C)$ revealed a great retention of the PF location for the Ref-4T group. Dotted lines represent chance level vs. chance level $* \star * P<0.001$. vs. target quadrant or $L P F$ zone ${ }^{\circ 0} P<0.001$. (D) Percentage of time ( \pm SEM) spent in the target quadrant relative to the nontarget quadrants (data averaged for the three nontarget quadrants) revealed that the Ref-2T group, unlike the Ref-4T group, did not display any preference for the target quadrant: $* \star \star P<0.001$.

curred during initial learning (Days 1 or 3 vs. Naïve: $P$ s $<0.01$ ) followed by a progressive decrease on Day 4 (vs. Naïve: $P<0.05$ ) to return to near baseline level on Day 9 (vs. Naïve: $P>0.1$ ). Post-hoc tests indicated significant differences for ST pCREB-ir between Days 1-4 and Day 9 (all Ps $<0.01$ ). Unlike the hippocampus and the ST, there was no evidence of any difference between trained and Naïve mice in the prelimbic cortex (PL; Fig. 3C) and the lateral nucleus of the amygdala (LA; Fig. 3D), except for pCREB-ir in the PL on Day 9, which was reduced relative to Naïve controls $(P<0.05)$.

\section{The Ref-4T task triggers differential temporal patterns of CREB phosphorylation in CAI and CA3 areas}

To determine the temporal dynamics of hippocampal CREB phosphorylation induced by the Ref-4T task, we measured pCREB-ir levels in the BT-Day 9 group trained for $8 \mathrm{~d}$ and then killed $24 \mathrm{~h}$ later just before the ninth session and in Ref-4T animals sacrificed on Day 9 at short $(0,15,60 \mathrm{~min})$ and long $(3,9$, 24, $48 \mathrm{~h}$ ) time intervals. pCREB-ir levels in hippocampal CA1 and CA3 areas were significantly increased after training on Day 9 (Fig. 4), whatever the time intervals examined (vs. Naïve: all Ps $<0.001)$. Interestingly, the BT-Day 9 group also displayed a

A

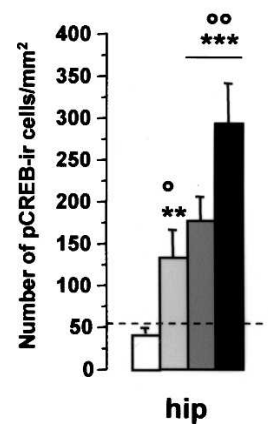

B

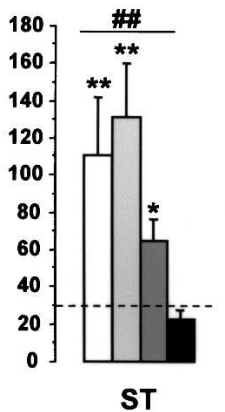

C

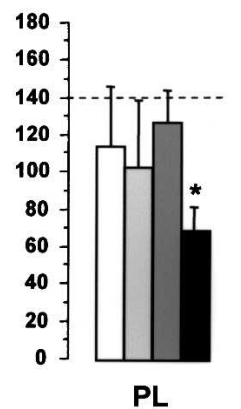

highly significant increase in pCREB-ir levels in both CA1 and CA3 areas (both $P \mathrm{~s}<0.0001$ ), suggesting that hippocampal CREB phosphorylation remained significantly elevated for at least $24 \mathrm{~h}$ after training on Day 8. Thus, to examine the kinetics of pCREB-ir specifically related to the ninth training session, we directly compared the values obtained in trained mice sacrificed at the different time intervals after Day 9 to those of the BT-Day 9 group. When compared to the BT-Day 9 group, mice subjected to the ninth training session displayed differential temporal patterns of pCREB-ir in CA1 and CA3 areas (Fig. 4A,B, respectively). ANOVA revealed a main effect of Subfield $\left(F_{(1,53)}=54.85 ; P<0.0001\right)$, Delay of sacrifice $\left(F_{(7,53)}=11 ; P=0.0001\right)$, and interaction $\left(F_{(7,53)}=4.79 ; P=0.001\right)$. Interestingly, CA1 pCREB-ir was significantly increased immediately and $15 \mathrm{~min}$ after training (vs. BTDay 9: $P<0.001$ and $P<0.01$, respectively; Fig. 4A,C). A highly significant increase in CA1 pCREB-ir was also observed at a 9-h time interval (BT-Day 9: $P<0.001$ ), suggesting that training triggered a biphasic pattern in CREB phosphorylation in CA1. In CA3, pCREB-ir significantly increased immediately until $60 \mathrm{~min}$ after training (vs. BT-Day 9: $P<0.01$ for $0-15 \mathrm{~min} ; P<0.05$ for 60 min). Nevertheless, at later time intervals, CA3 pCREB-ir returned to near BT group level, suggesting an early transient learning-related CREB phosphorylation. Confirming the Ref-4T learning-induced CREB phosphorylation sustained for up to $24 \mathrm{~h}$, pCREB-ir levels in both CA1 and CA3 areas from the $24 \mathrm{~h}$ - and $48 \mathrm{~h}$-Day 9 groups were significantly higher relative to those of Naïve controls (all $P s<0.001)$ and did not differ from the BT-Day 9 group (both $P$ s $>0.5$ ).

Extended training, but not early training, induces sustained $p C R E B$-ir and Zif268-ir in $C A 1$ and $C A 3$ areas

We next asked whether the sustained increase in hippocampal pCREB-ir found in the BT-Day 9 group could be associated with an up-regulation of target early genes. To address this question, we compared the levels of Zif268-ir in Naïve controls to those of trained mice sacrificed immediately prior to or $60 \mathrm{~min}$ af-
Figure 3. Differential learning-related changes in pCREB-ir during acquisition of the spatial reference memory task across four brain structures. Mean ( \pm SEM) number of pCREB immunoreactive cells (pCREB-ir) per $\mathrm{mm}^{2}$ for the Ref-4T groups sacrificed 60 min after training on Days 1, 3, 4, and 9 in $(A)$ hippocampal subfields (hip); (B) dorsal striatum (ST); (C) prelimbic nucleus of the prefrontal cortex (PL); and $(D)$ lateral nucleus of the amygdala (LA). Dotted lines indicate baseline levels of Naïve control mice vs. Naïve ${ }^{* *} P<0.01 ;{ }^{* \star *} P<0.001$ vs. Day $1{ }^{\circ} P<0.05 ;{ }^{\circ} P<0.01$ vs. Day $9 \# \# P<0.01$. 

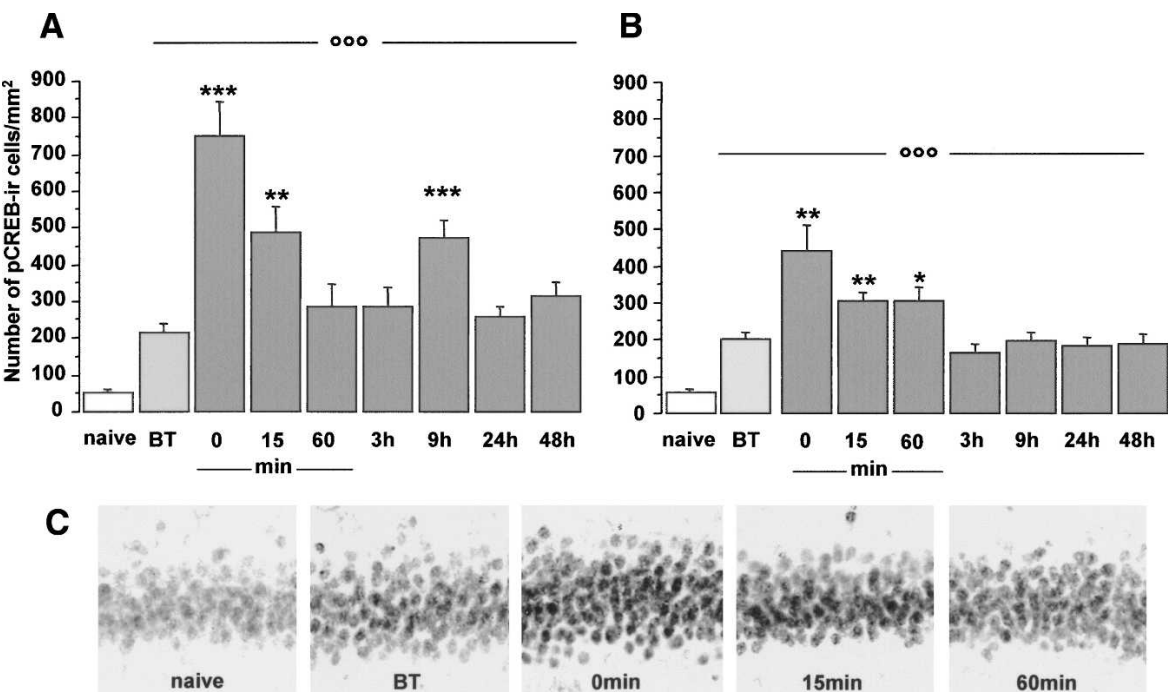

Figure 4. Differential patterns of CREB phosphorylation within hippocampal CA1 and CA3 areas. Mean number ( \pm SEM) of pCREB-ir per $\mathrm{mm}^{2}$ in CA1 $(A)$ and CA3 $(B)$ areas. The differential patterns of pCREB-ir were examined in mice immediately prior to training (BT group) or at seven time points after final probe trial on Day 9 vs. BT group ${ }^{*} P<0.05 ;{ }^{* *} P<0.01 ;{ }^{* \star *} P<0.001$; vs. Naïve ${ }^{000} P<0.001$. (C) Representative photomicrographs illustrating CA1 pCREB immunoreactivities in the two control groups (Naïve and BT-Day 9) and Ref-4T animals sacrificed at either 0, 15, or 60 min after training on Day 9.

ter training on Day 9 (Fig. 5). Levels of Zif268-ir in CA1 and CA3 areas were significantly elevated in both trained groups (vs. Naïve controls: $+70 \%-80 \%$; Ps $<0.05$; Fig. $5 \mathrm{~A}$ ). Since mice were subjected to repeated training over several daily sessions, it was possible that CREB and Zif268 may have been differentially activated as a function of "early" or "late" training sessions. We thus compared pCREB-ir and Zif268-ir levels in CA1 and CA3 areas of mice sacrificed immediately prior to training on Day 4 (early training) or Day 9 (late training) sessions. In the BT-Day 4 group, Zif268-ir (Fig. 5A) and pCREB-ir (Fig. 5B) levels did not differ from those of Naïve controls $(P s>0.2)$ and were significantly lower than those found in BT-Day 9 animals $(P \mathrm{~s}<0.01)$. This suggests that sustained hippocampal pCREB-ir and Zif268-ir for up to $24 \mathrm{~h}$ occurred specifically after extended training.

\section{The biphasic pattern of CREB phosphorylation occurs specifically in CAl area}

To determine whether the biphasic pattern of CREB phosphorylation was specific to the CA1 area, we examined pCREB-ir levels in other brain structures (LA, PL, and ST) from the BT-Day 9 group and four Ref- $4 \mathrm{~T}$ groups sacrificed immediately, $15 \mathrm{~min}, 60$ min, or $9 \mathrm{~h}$ after Day 9. Completion of the Ref-4T task triggered a very different pattern of pCREB-ir in LA, PL, and ST (Fig. 6). In contrast to CA1 CREB staining, no differences occurred for the three other regions between the BT-Day 9 and Naïve controls (all $P$ s $>0.2$ ). In the PL and LA, pCREB-ir levels were significantly increased immediately after the ninth training session (vs. Naïve or BT-Day 9: all Ps < 0.01) but returned to near BT-Day 9 level at later time intervals. In contrast, there were no differences for ST pCREB-ir between trained and Naïve or BT-Day 9 groups, whatever the time interval examined $(P>0.1)$.

\section{The duration of the early peak of CAI CREB phosphorylation differs in the Ref-4T and Ref-2T groups}

We next examined whether the early peak of CA1 CREB phosphorylation was specific to mice that had acquired the Ref-4T task or whether it could also occur when the reference memory was not yet consolidated (Ref-2T group; see Fig. 2A-D). As shown

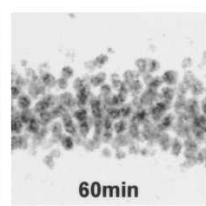

in Figure 7A, the duration of CA1 CREB phosphorylation differs depending on the training protocol. Relative to respective Ref-4T groups, CA1 pCREB-ir levels were significantly reduced in the Ref-2T group at 15 min post-training on Day 9 $(P<0.05)$. One-way ANOVA on CA1 pCREB data from Ref-2T groups revealed a significant main effect of Delay of sacrifice $\left(F_{(3,21)}=8.22 ; P<0.001\right)$. CA1 pCREB-ir significantly increased immediately after Ref-2T training on Day 9 (vs. Naïve or BT-Ref-2T: Ps $<0.001$ ) but returned near BT-Ref-2T levels $(P s>0.2)$ at $15-60-$ min time intervals. These results raise the possibility that learningrelated changes in CA1 pCREB-ir observed at the 15-min time interval might be directly related to spatial learning performance. Correlation analyses were thus performed between changes in CA1 pCREB-ir and individual learning performance during the probe test. When the 15 min-Ref-2T group was considered, CA1 pCREB-ir levels were negatively related to the percentage of time spent in the LPF zone $(r=-0.741$; $P=0.035 ;$ Fig. $7 \mathrm{~B})$. In contrast, there was no statistically reliable correlation for the Ref-2T groups at other time intervals. Similar analyses conducted on data from the Ref-4T groups indicated that, whatever the time interval considered, no statistically reliable correlation existed between spatial learning performance and CA1 pCREB levels.

\section{Discussion}

The main findings of this study are: (1) Acquisition of the reference memory task induced significant changes in CREB phosphorylation, which varied as a function of both the brain structures considered and the degree of learning. Namely, training in the spatial reference task was associated with progressively increasing levels of pCREB-ir in the dorsal hippocampus, whereas CREB phosphorylation in the striatum significantly increased on Day 1 but then decreased with further training; (2) spatial reference memory consolidation triggered a biphasic CREB phosphorylation in hippocampal CA1, whereas a sole early peak of CREB phosphorylation was observed in CA3 and other structures; (3) persistent CREB phosphorylation and Zif268 up-regulation were found in hippocampal CA1 and CA3 areas after extensive training (BT-Day 9) but were absent during early training (BT-Day 4); (4) a strong CA1 CREB phosphorylation was observed immediately after training irrespective of acquisition of the behavior. In contrast, at $15 \mathrm{~min}$ after training, the changes in the CA1 CREB phosphorylation state were specifically related to individual behavioral performance. The present results suggest that hippocampal-learning specificity of CREB may be reflected best by duration, rather than magnitude, of CREB phosphorylation.

\section{Behavior}

To address the relationship between hippocampus-dependent memory and CREB activation, we examined the levels of pCREBir in young-adult $\mathrm{C} 57 \mathrm{Bl} / 6$ mice trained in a water maze reference memory task. Acquisition of the reference memory requires the hippocampus to form spatial associations between the PF location and the surrounding environment, making the hippocampus necessary for this task (Morris et al. 1982; Sutherland et al. 
A
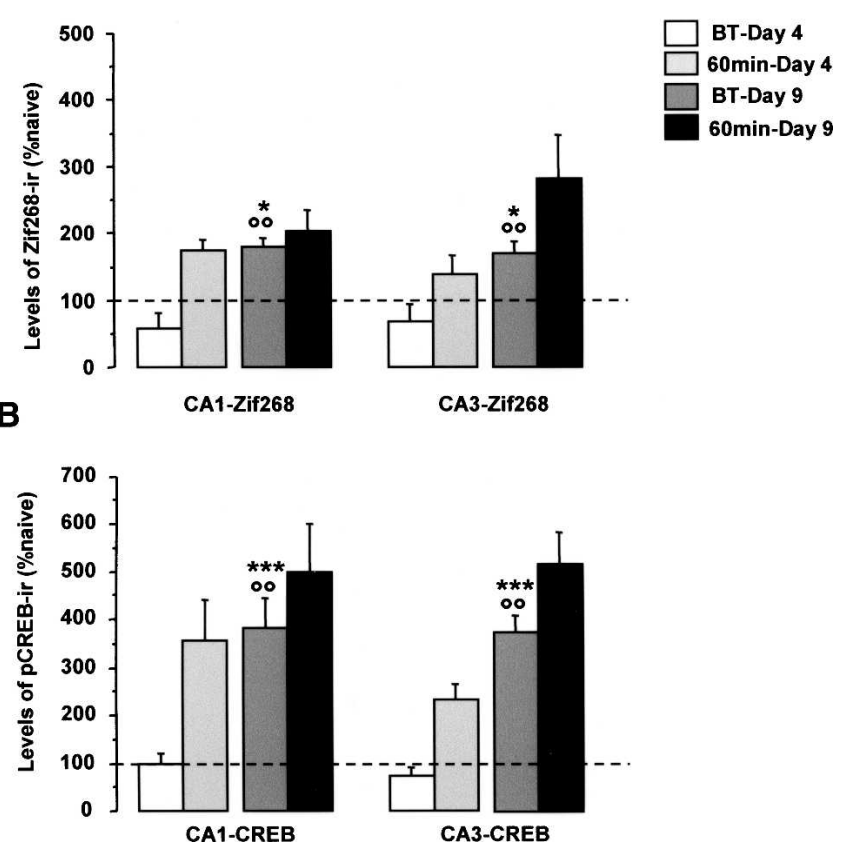

Figure 5. Extended training triggers sustained CREB phosphorylation and Zif268 up-regulation in the hippocampus. Levels of Zif268-ir $(A)$ and pCREB-ir (B) were examined in the following groups: BT-Day 4; BT-Day 9; 60 min-Day 4; and 60 min-Day 9. Data for Zif268 and pCREB-ir in CA1 (left) and CA3 (right) areas were normalized to those of Naive controls. BT-Day 9 vs. BT-Day $4{ }^{\circ 0} P<0.01$; BT-Day 9 vs. Naïve ${ }^{*} P<0.05$; $* * * P<0.001$.

2001; Roozendaal et al. 2004). The training in a spatial-spaced protocol of four daily trials/session over $9 \mathrm{~d}$ produced long-term spatial memory. All Ref-4T animals were highly selective with regard to their PF area preference during the probe test on Day 9. When compared to the Ref-4T group, the Ref-2T animals showed a delayed acquisition as assessed by higher escape latencies to reach the PF from Day 3 to Day 9. Furthermore, during the final probe test, they failed to display a good retention of the PF location, as revealed by no preferences for the target quadrant. This led us to conclude that memory for the PF location may not yet be consolidated in the Ref-2T group.

\section{Acquisition of the spatial reference task was associated} with region-specific patterns of CREB phosphorylation A post-training phosphorylation of CREB has been implicated in memory formation of spatial learning tasks in rodents (Mizuno et al. 2002; Colombo et al. 2003; Martel et al. 2007; Porte et al. 2008). Our results show that acquisition of the reference memory task is associated with significant changes in pCREB levels which varied as a function of both the regions considered and the degree of learning. When pCREB-ir was examined $60 \mathrm{~min}$ after training, acquisition of the reference memory task was accompanied by a progressive increase of pCREB-ir in the dorsal hippocampus. The learning-related increase in hippocampal pCREB-ir occurred when animals began to display above-chance discrimination performance (Days 3 and 4) and reached maximal level on the final training session (Day 9). It has been recently shown that CREB activation in the dorsal CA1 region depends on the interaction between the learning demands and stress (Kogan and Richter-Levin 2008). Because there was no difference in the levels of hippocampal pCREB-ir between the Ref-4T group sacrificed on Day 1 and Naïve caged controls, it is unlikely that a generalized response to swimming stress or novelty may have a major impact on the changes of CREB phosphorylation found in the hippocampus. Our results concur with previous findings in rats reporting that the radial arm maze training results in a significant increase in PKA activity and CREB phosphorylation in the hippocampus in the course of spatial learning (Mizuno et al. 2002). These data are also in agreement with all the previous works from many laboratories demonstrating that hippocampal CREB activation plays a key role in the formation and stabilization of spatial LTM (Bernabeu et al. 1997; Silva et al. 1998; Cammarota et al. 2000). Moreover, blockade of hippocampal CREB function in CA1 or CA3 regions, using either antisense (Guzowski and McGaugh 1997; Florian et al. 2006) or overexpression of dominant negative CREB (Pittenger et al. 2002), results in a deficiency in spatial long-term, but not short-term, memory in the water maze, whereas increasing levels of hippocampal CREB protein during spatial training facilitates formation of LTM (Brightwell et al. 2007).

In contrast to the progressive increase in pCREB-ir seen in the dorsal hippocampus, CREB phosphorylation in the striatum was strongly increased at the earliest stages of acquisition (first and third sessions) but decreased as learning progressed (fourth and ninth sessions) to return to near baseline level. Our findings suggest that CREB-dependent signaling cascade underlying spatial memory formation might be oppositely regulated in the hippocampal and striatal regions. The opposite patterns of CREB phosphorylation observed in the two regions during the course of spatial learning are interesting, in light of previously reported evidence for functional dissociations between these two structures (Packard et al. 1989, 1994; Packard and McGaugh 1992; Packard and Teather 1997). Further, a previous study has shown that, at 60 min post-training, cross maze training that could be solved using either a response or a place strategy resulted in increased CREB phosphorylation either in the hippocampus for "place learners" or in the striatum of "response learners" (Colombo et al. 2003). Also, differential inductions of immediate early genes (c-Fos and c-Jun) have been observed in the rat hippocampus and dorsal striatum after training in cued versus spatial tasks (Teather et al. 2005; Gill et al. 2007). Interestingly, two

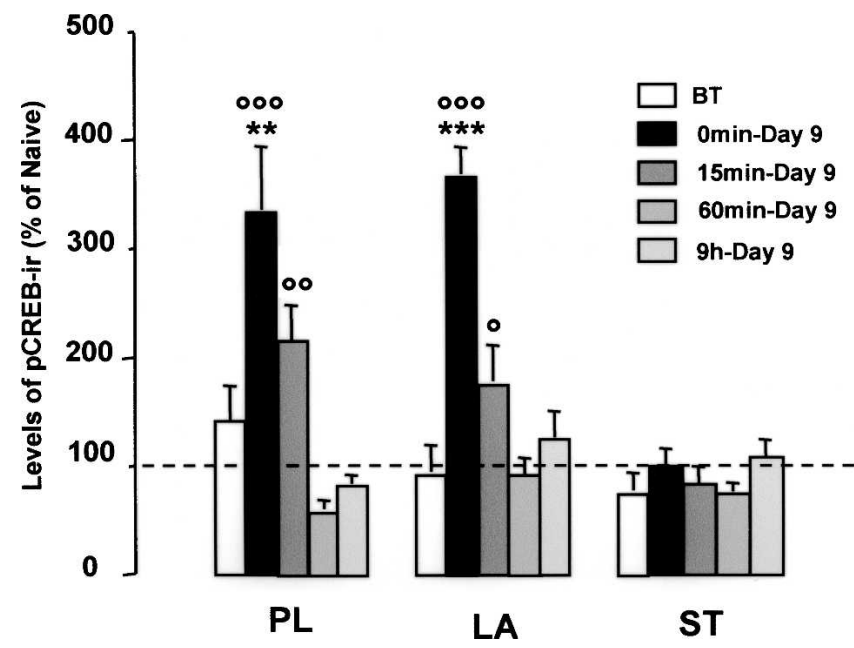

Figure 6. Region-specific patterns of CREB phosphorylation associated with spatial training in the Ref-4T task. $(A)$ The learning-related changes in pCREB-ir were determined in three structures related to the hippocampus in trained mice sacrificed either immediately prior to (BT-Day 9) or 0, 15, $60 \mathrm{~min}$, and $9 \mathrm{~h}$ after training on Day 9. Data were normalized to those of Naïve controls. PL, prelimbic cortex; LA, lateral amygdale; and ST, dorsal striatum vs. Naïve ${ }^{\circ} P<0.05 ;{ }^{\circ 0} P<0.01 ;{ }^{\circ 0} P<0.001$ vs. BTDay $9{ }^{* *} P<0.01 ;{ }^{* *} P<0.001$. 
A

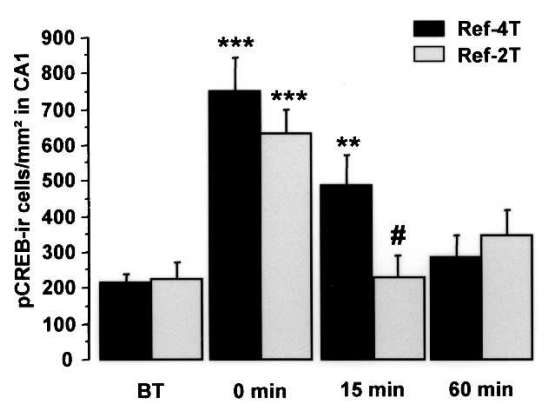

B

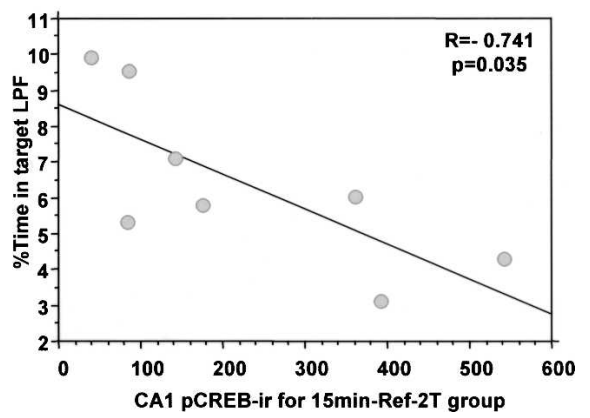

Figure 7. Relationships between early patterns of CA1 CREB phosphorylation and behavioral performance. Comparisons of CA1 pCREB-ir levels in Ref-4T and Ref-2T animals sacrificed immediately prior to (BT-Day 9) or 0,15 , and 60 min after training on Day 9. (A) Mean ( \pm SEM) number of pCREB-ir cells per $\mathrm{mm}^{2}$. (B) Relationships between CA1 pCREB-ir levels of Ref-2T mice sacrificed at 15-min time interval and individual performance during probe test on Day 9. vs. respective BT-Day $9{ }^{* \star} P<0.01$; ${ }^{* * *} P<0.001 ;$ vs. $15 \mathrm{~min}-\mathrm{Ref}-4 \mathrm{~T} \# P<0.05$.

recent studies in rats (Broadbent et al. 2007) and mice (Martel et al. 2007) have demonstrated that memory tasks that depend on the hippocampus (two-choice discrimination task and spatial water maze task, respectively) are primarily supported by the dorsal striatum (and habit-based memory strategy). Collectively, these findings suggest that, during the course of spatial learning, the striatum may be involved for initial acquisition and then a shift would occur from the striatum to the hippocampus with increased CREB phosphorylation within the hippocampus being functionally decisive for improvement and stabilization of learning performance.

\section{Spatial reference memory led to a biphasic pattern of CREB phosphorylation in hippocampal CAl area}

Following completion of the ninth training session of the Ref-4T task, two peaks of pCREB-ir were detected in the hippocampal CA1 area at $0-15-$ min and at 9 -h post-training time intervals. Then, CA1 pCREB-ir levels decreased progressively at later time intervals (i.e., 24 and $48 \mathrm{~h}$ ) but remained well above those of Naïve caged controls. The biphasic pattern of CREB phosphorylation induced by spatial learning in CA1 area is in agreement with earlier studies showing that consolidation of hippocampusdependent learning tasks involves at least two waves of neuronal plastic changes (Bernabeu et al. 1997; Taubenfeld et al. 1999; Cammarota et al. 2000; Stanciu et al. 2001; Bilang-Bleuel et al. 2002; Colombo et al. 2003; Martel et al. 2007). In addition, Schulz and colleagues found that induction of late long-term potentiation in the dentate gyrus of rats evokes a biphasic response of CREB phosphorylation (Schulz et al. 1999). We recently demonstrated that contextual fear memory consolidation, which is hippocampus dependent, requires two phases of CA1 ERK/CREB activation, whereas cue conditioning consolidation, which is amygdala dependent, is associated with a transient early phase of hippocampal ERK/CREB activation (Trifilieff et al. 2006). We and others have proposed that the development of one or two waves of neuronal plasticity likely represents a general mechanism that depends on the neuropsychological characteristics of the learning protocol (Bourtchuladze et al. 1994; Swank and Sweatt 2001; Trifilieff et al. 2006, 2007). Here, the differential temporal dynamics of CREB phosphorylation observed in CA1 (biphasic) and CA3 (monophasic) areas are consistent with previous findings reporting differential hippocampal subfields engagement according to the demands and the degree of mastery of a spatial task. In particular, this is consistent with the notion that CA3 may be important for the encoding of spatial information, whereas CA1 may rather be engaged in the maintenance of spatial representations and consolidation (Brun et al. 2002) or retrieval processes (Kesner et al. 2004; Remondes and Schuman 2004; Poirier et al. 2008).

Our findings also indicate that extensive training in the reference task procedure triggered persistent CREB phosphorylation that lasted for up to 24 $\mathrm{h}$ in the hippocampal CA1 and CA3 areas. An elevated hippocampal CREB phosphorylation, for up to $24 \mathrm{~h}$, has also been previously described after inhibitory avoidance learning (Taubenfeld et al. 1999, 2001) and contextual fear conditioning (Trifilieff et al. 2006). Strikingly, we found that (1) significant CREB phosphorylation at the 24-h time interval was restricted to hippocampal neurons and (2) occurred after extended training in the Ref-4T task (BT-Day 9 group) but not during initial learning (BT-Day 4 group). Together with the fact that elevated hippocampal pCREB-ir in the BT-Day 9 group was tightly associated with an up-regulation of Zif268-ir, this suggests that sustained CREB activation and Zif268 induction for up to $24 \mathrm{~h}$ after training may act as critical components for maintaining and updating earlier memory traces in hippocampal networks. Consistent with our findings, a similar up-regulation of c-Fos and Zif268 by protein synthesis- and BDNF-dependent events involved in LTM persistence was recently shown by Bekinschtein et al. (2007). Further, a robust Arc induction was also observed after repeated 24-h distant exposures to the same environment or even during overtraining in a spatial task (Guzowski et al. 2001, 2006). Among the protein kinases that can be responsible for sustained CREB phosphorylation after learning, prolonged ERK activation appeared to be necessary for maintaining learninginduced cellular modifications for days after training (CohenMatsliah et al. 2007). Further, previous studies reported that multiple-trial conditioning that induced LTM also resulted in an extremely prolonged PKC and PKA activation in honeybee (Grunbaum and Muller 1998; Muller 2000).

In the present study, pCREB-ir levels were highly significantly increased immediately and $15 \mathrm{~min}$ after the probe test on Day 9, relative to the BT-Day 9 group. A learning-related increase in pCREB-ir occurred immediately after training in the hippocampus, the LA, and the PL, whereas by 15 min post-training, pCREB-ir remained significant in hippocampal areas, but not in other regions. The findings that CREB phosphorylation at up to a 15-min time interval was restricted to the hippocampus confirmed and extended previous studies suggesting that regionallearning specificity of CREB may be reflected best by sustained, rather than immediate, CREB activation (Stanciu et al. 2001; Colombo et al. 2003).

Interestingly, a strong increase in CA1 pCREB was also observed immediately after training in the Ref-2T group, supporting our hypothesis that immediate CA1 CREB phosphorylation was not related strictly to learning (Ref-4T mice had already reached asymptotic performance) or to memory (Ref-2T mice did not express a good memory on the probe trial). By 15 min after training, CA1 pCREB returned to the baseline level in the Ref-2T animals but remained significantly elevated in the Ref-4T group. This suggests again that hippocampal-learning specificity of CREB may be reflected best by duration, rather than amplitude, of CREB phosphorylation. Although CA1 pCREB levels were 
highly significantly increased in the Ref-4T mice sacrificed immediately and $15 \mathrm{~min}$ after the probe test, no correlation was observed between behavioral performance and changes in CA1 pCREB at any time of sacrifice. The present findings confirm and extend previous observations indicating that there was no correlation between CA1 pCREB-ir and performance for 129T2/Sv young-adults trained in a similar Ref- $4 \mathrm{~T}$ task over $3 \mathrm{~d}$, whereas deficits in pCREB levels were associated to memory impairments in aged animals (Porte et al. 2008). As all Ref-4T animals had reached asymptotic performance, we can prudently suggest that the high performance and narrow range of scores observed during probe trial in the Ref- $4 \mathrm{~T}$ animals may have interfered with the reasonable ability to see a correlation between the levels of CREB phosphorylation and memory performance. In contrast, a significant and negative correlation between CA1 pCREB levels and performance on the probe trial was observed in the $15 \mathrm{~min}$ Ref-2T group. The present finding that Ref-2T animals with the best recall of PF location tended to have the lowest CA1 pCREB suggests that increased pCREB levels in the 15min-Ref-2T group probably reflect a generalized nonspecific activation of the dorsal hippocampus (i.e., exposure to the training procedure) and not the capability of animals to learn (i.e., where to find the PF location). This result might be related to those of Moncada and Viola (2006) who recently showed that bidirectional change of the phosphorylation state of CREB in the rat hippocampus is associated with exposure to a novel detection or to a familiar environment. Other factors to consider for the high pCREB levels in poor Ref-2T learners include alterations of the fine-tuning of CREB-mediated transcriptional responses, a condition that has detrimental effects on the learning processes. It has been proposed that, a few days after learning completion, a selective, network-specific process of translational and transcriptional events is required for allowing both spatial memory to be fine-tuned and maintained (Bekinschtein et al. 2007; Miyashita et al. 2008) and persistence of long-lasting learning-induced enhancement of neuronal excitability (Taubenfeld et al. 2001; Abraham et al. 2002; Ramirez-Amaya et al. 2005; Brightwell et al. 2008). Thus, our findings showing a robust increase of CA1 pCREB-ir levels in the Ref-4T mice trained for $9 \mathrm{~d}$ suggests that a selective recruitment of a CREB-dependent pathway in hippocampal CA1 region in the following days and/or week(s) after learning completion may be required to make memory traces stronger and more stable.

In conclusion, the present findings provide evidence for spatial learning-induced changes in CREB phosphorylation in the hippocampus of mice. The findings here and elsewhere (Mizuno et al. 2002; Moncada and Viola 2006; Brightwell et al. 2007) support the hypothesis that CREB phosphorylation in the hippocampus is a critical step for spatial memory formation. The present data indicate that the direction (increase in the hippocampus vs. decrease in the striatum), the localization (CA1 vs. other brain regions), and the dynamics (biphasic vs. monophasic) of CREB phosphorylation must be modulated within a narrow window for efficient acquisition and consolidation of spatial memory. Further experiments are currently being carried out to examine this question in detail.

\section{Materials and Methods}

\section{Animals}

A total of 119 male mice of the C57BL/6 strain (8 wk old and 25-35 g at arrival; Iffa Credo) was used in this study. They were maintained on a 12-h light-dark artificial cycle (lights on at 7:00 a.m.), in a temperature-controlled colony room $\left(22 \pm 1^{\circ} \mathrm{C}\right)$ and were provided with food and water ad libitum. One week before behavioral training, animals (20 wk old) were individually housed and handled each day for a duration of $5 \mathrm{~min}$. They were then tested in the water maze during the light phase between 7:30 and 18:00. All experimental procedures were conducted in accordance with the European Communities Council Directive of 24 November 1986 (86/609/EEC).

\section{Apparatus}

The water maze consisted of a swimming pool based on that described by Morris (1984) and adapted for mice. It consisted of a circular tank (140 $\mathrm{cm}$ diameter, $40 \mathrm{~cm}$ high), filled to a depth of $30 \mathrm{~cm}$ with water maintained at $20^{\circ} \mathrm{C}$ and rendered opaque by the addition of white nontoxic paint (Pebeo). The pool was located in a room uniformly illuminated by a halogen lamp and equipped with various distal cues. Located inside the pool was a removable, circular (13 cm diameter) platform (PF) made of transparent Plexiglas, positioned such that its top surface was 0.5 $\mathrm{cm}$ below the water. Data were collected using a video camera fixed to the ceiling of the room and connected to a video recorder and to a video-tracking system (videotrack, Viewpoint) detailed in Malleret et al. (1999), both located in an adjacent room, which housed the home cages of the mice undergoing testing.

\section{Behavioral procedures}

\section{Pretraining}

Two days prior to the acquisition phase, mice performed a familiarization session in order to acquire the procedural aspects of the tasks (see Malleret et al. 1999). It consisted of placing the subject on the PF occupying a central position for $15 \mathrm{~s}$, and then releasing it from two peripheral points and verifying that it could swim and climb on the PF.

\section{General training procedure}

On each day, mice were subjected to one session of four trials (Ref-4T group). A trial consisted of placing the mouse into the water facing the outer edge of the pool in one of the virtual quadrants (except the quadrant where the PF was located) and allowing it to escape to the submerged PF. A trial terminated when the animal reached the PF where it was allowed to remain for $15 \mathrm{~s}$. If the animal failed to find the target before $90 \mathrm{~s}$ during the earlier stages of learning, it was manually guided to the PF where it was allowed to stay for $15 \mathrm{~s}$. After completion of a trial, mice were removed from the pool and placed back to their home cages in the adjacent room, beneath heat lamps in order to reduce the loss of core temperature. An additional group of mice performed only two trials per session for nine days (Ref-2T group). For each day of sacrifice, session durations were similar for Ref-4T and Ref-2T groups with intertrial intervals of $2 \mathrm{~min}$.

\section{Experimental protocol}

\section{Ref-4T groups}

Mice were trained to reach the PF, which occupied a constant location (the center of the southwest quadrant) from three peripheral releasing points (northwest, northeast, and southeast), according to a counterbalanced sequence of four successive trials per day with an intertrial interval of 2 min. The experimental design of the present study is summarized in Figure 1. To examine whether acquisition of the reference memory task was associated with region-specific changes in pCREB-ir, trained mice were sacrificed $60 \mathrm{~min}$ after the final trial on Days $1(N=5), 3$ $(N=5), 4(N=8)$, and $9(N=8)$. In order to assess the memory for the PF location after the ninth session, each mouse performed a probe test (90 s, PF removed) immediately after the last training trial. To study the time course and persistence of CREB phosphorylation following the acquisition of the reference learning task, levels of pCREB-ir were examined in mice sacrificed at seven different time points after probe test on Day 9: $0 \mathrm{~min}(N=5), 15$ min, $60 \mathrm{~min}, 3 \mathrm{~h}, 9 \mathrm{~h}, 24 \mathrm{~h}$, and $48 \mathrm{~h}$ ( $N=8$ for each). To control for a possible long-lasting CREB phosphorylation once the task was well established, a group of mice was trained for $8 \mathrm{~d}$ and then killed $24 \mathrm{~h}$ later just before the ninth session (BT-Day 9 group; 
$N=8$ ). Finally, to study whether spatial training could be associated with long-lasting CREB phosphorylation and Zif268 activation during initial acquisition learning, another control group of mice was sacrificed $24 \mathrm{~h}$ after the third training session immediately prior to training on Day 4 (BT-Day 4 group; $N=5$ ).

\section{Ref-2T groups}

Mice were submitted to two daily trials over nine consecutive days of the reference learning task. The PF location was constant over the whole training period. Mice were sacrificed just before the ninth training session $(N=6)$ or at $0 \min (N=5), 15 \mathrm{~min}$ $(N=8)$, or $60 \min (N=6)$ after probe test on Day 9.

\section{Naïve control group}

Undisturbed mice (Naïve; $N=10$ ) were handled similarly to other trained animals prior to the acquisition phase, and taken out of their home cages to be sacrificed altogether with the Ref$4 \mathrm{~T}$ and Ref-2T groups.

\section{Quantification and analysis of behavioral data}

The movements of the subjects were recorded using the videotrack system as previously described (detailed in Malleret et al. 1999). The data were processed using Excel (Microsoft), and analyzed for each acquisition trial as (1) the latency, i.e., the time (in seconds) spent by mice to escape from the releasing point to the submerged PF and (2) the mean swim speed (path length/latency, in centimeter/second). A measure was taken every $40 \mathrm{~ms}$ of the whole swim path then averaged. Group (Ref-4T and Ref-2T) was the between-subject factor, and Day and Trial were the main within-subject factors for the analyses of variance (using StatView 5.01, SAS Institute Inc.). Further analyses of individual group comparisons were used when the effect of the main factors or their interaction was significant $(P<0.05)$.

Analyses were also conducted for the probe trials in terms of the relative time spent in the target quadrant or large PF area (LPF, $26 \mathrm{~cm}$ diameter, centered on the target PF area), by using the following percentages: $100 \times$ (time in the target quadrant/ time in the whole pool), and $100 \times$ (time in the target LPF area/ time in the whole pool). ANOVAs were performed on these data with the group and zone (target vs. nontarget) as the main factors. Finally, data on target quadrant and LPF zones were compared (Student's $t$-test) to their respective chance level for swimming in one quadrant zone (i.e., $25 \%=100 \times$ area of one quadrant/whole pool area $\left[\pi \times 70^{2}\right]$ ), or one LPF zone (i.e., $3.45 \%=100 \times$ area of the LPF zone $\left[\pi \times 13^{2}\right] /$ whole pool area).

\section{Immunohistochemical procedures}

At the appropriate time interval (see Fig. 1), mice were deeply anaesthetized and perfused transcardially with ice-cold $4 \%$ paraformaldehyde in $0.1 \mathrm{M}$ phosphate buffer $(\mathrm{PB})$. The brains were removed and stored overnight in the same fixative solution, sectioned $(50 \mu \mathrm{m})$ on a vibratome (Leica) and then kept in a solution containing $30 \%$ ethylene glycol, $30 \%$ glycerol, $0.1 \mathrm{M} \mathrm{PB}$ at $-20^{\circ} \mathrm{C}$ until processed for immunohistochemistry. All solutions contained the phosphatase inhibitor sodium fluoride $(\mathrm{NaF} ; 0.25$ $\mathrm{mM})$. Free-floating sections were rinsed in $0.1 \mathrm{M}$ Tris buffer saline (TBS, $\mathrm{pH} 7.4$ ), incubated with $0.5 \% \mathrm{H}_{2} \mathrm{O}_{2}$ in TBS to inhibit endogenous peroxidase. After blocking in TBS containing 1\% bovine serum albumin, 3\% normal goat serum, and $0.2 \%$ Triton $\mathrm{X}-100$, tissue sections were incubated at $4^{\circ} \mathrm{C}$ for $48 \mathrm{~h}$ with rabbit primary polyclonal antibody that recognizes the active/ phosphorylated form of CREB (anti-pCREB, 1:2000, Upstate Biotechnology) or a rabbit polyclonal anti-Zif268 antibody (1:5000, Santa Cruz Biotechnology: sc-110). Incubation with a biotinylated goat anti-rabbit IgG (1:2000; Jackson Immunoresearch) for 2 $\mathrm{h}$ at room temperature was followed by amplification with avidin-biotinylated horseradish peroxidase complex (Vectastain Elite kit, Vector Laboratories) for $2 \mathrm{~h}$. The staining was then visualized by using diaminobenzidine. The reaction was stopped by washing in ice-cold $\mathrm{TB}$ and then sections were mounted on gelatin-coated slides, dehydrated, and coverslipped.

\section{Quantification and analysis of immunohistochemical data}

Quantitative analysis was performed using an imaging analysis system (Biocom Visiolab 2000, V4.50). The experimenter was blind to the experimental groups of mice examined. For all region analyses, counts were taken bilaterally with a $10 \times$ objective from at least three consecutive sections per animal (the rostrocaudal distance between consecutive sections was $0.2 \mathrm{~mm}$ ) and the number of positive nuclei per $\mathrm{mm}^{2}$ was averaged to produce a mean. The hippocampal subfields and other brain regions were identified from coronal sections, using the nomenclature of Franklin and Paxinos (1997). Quantification of pCREB-immunoreactive neurons (pCREB-ir) was made in CA1 and CA3 areas of the dorsal hippocampus (hip), the lateral nucleus of the amygdala (LA), the prelimbic part of the prefrontal cortex (PL), and the dorsal part of the striatum (ST). Immunohistochemical data were expressed as mean \pm SEM and analyzed by ANOVA (StatView statistical software) followed by appropriate post-hoc tests. In all cases, significance of results was accepted at $P<0.05$.

\section{Acknowledgments}

This work was supported by the Centre National de la Recherche Scientifique and Université de Bordeaux 1. We thank L. Decorte and A. Faugère for their technical assistance and D. Panzeri, N. Argenta, and J. Huard for their help in animal breeding and care.

\section{References}

Abraham, W.C., Logan, B., Greenwood, J.M., and Dragunow, M. 2002. Induction and experience-dependent consolidation of stable long-term potentiation lasting months in the hippocampus. $J$. Neurosci. 22: 9626-9634.

Balschun, D., Wolfer, D.P., Gass, P., Mantamadiotis, T., Welzl, H., Schutz, G., Frey, J.U., and Lipp, H.P. 2003. Does cAMP response element-binding protein have a pivotal role in hippocampal synaptic plasticity and hippocampus-dependent memory? J. Neurosci. 23: 6304-6314.

Bekinschtein, P., Cammarota, M., Igaz, L.M., Bevilaqua, L.R., Izquierdo, I., and Medina, J.H. 2007. Persistence of long-term memory storage requires a late protein synthesis- and BDNF-dependent phase in the hippocampus. Neuron 53: 261-277.

Bernabeu, R., Bevilaqua, L., Ardenghi, P., Bromberg, E., Schmitz, P., Bianchin, M., Izquierdo, I., and Medina, J.H. 1997. Involvement of hippocampal cAMP/cAMP-dependent protein kinase signaling pathways in a late memory consolidation phase of aversively motivated learning in rats. Proc. Natl. Acad. Sci. 94: 7041-7046.

Bilang-Bleuel, A., Rech, J., De Carli, S., Holsboer, F., and Reul, J.M. 2002. Forced swimming evokes a biphasic response in CREB phosphorylation in extrahypothalamic limbic and neocortical brain structures in the rat. Eur. J. Neurosci. 15: 1048-1060.

Bourtchuladze, R., Frenguelli, B., Blendy, J., Cioffi, D., Schutz, G., and Silva, A.J. 1994. Deficient long-term memory in mice with a targeted mutation of the cAMP-responsive element-binding protein. Cell 79: 59-68.

Bozon, B., Davis, S., and Laroche, S. 2003a. A requirement for the immediate early gene zif268 in reconsolidation of recognition memory after retrieval. Neuron 40: 695-701.

Bozon, B., Kelly, A., Josselyn, S.A., Silva, A.J., Davis, S., and Laroche, S. 2003b. MAPK, CREB and zif268 are all required for the consolidation of recognition memory. Philos. Trans. R. Soc. Lond. B Biol. Sci. 358: 805-814.

Brightwell, J.J., Smith, C.A., Neve, R.L., and Colombo, P.J. 2007. Long-term memory for place learning is facilitated by expression of cAMP response element-binding protein in the dorsal hippocampus. Learn. Mem. 14: 195-199.

Brightwell, J.J., Smith, C.A., Neve, R.L., and Colombo, P.J. 2008. Transfection of mutant CREB in the striatum, but not the hippocampus, impairs long-term memory for response learning. Neurobiol. Learn. Mem. 89: 27-35.

Broadbent, N.J., Squire, L.R., and Clark, R.E. 2007. Rats depend on habit memory for discrimination learning and retention. Learn. Mem. 14: $145-151$.

Brun, V.H., Otnass, M.K., Molden, S., Steffenach, H.A., Witter, M.P., Moser, M.B., and Moser, E.I. 2002. Place cells and place recognition maintained by direct entorhinal-hippocampal circuitry. Science 296: 2243-2246.

Cammarota, M., Bevilaqua, L.R., Ardenghi, P., Paratcha, G., Levi de 
Stein, M., Izquierdo, I., and Medina, J.H. 2000. Learning-associated activation of nuclear MAPK, CREB and Elk-1, along with Fos production, in the rat hippocampus after a one-trial avoidance learning: Abolition by NMDA receptor blockade. Brain Res. Mol. Brain Res. 76: 36-46.

Cammarota, M., Bevilaqua, L.R., Kohler, C., Medina, J.H., and Izquierdo, I. 2005. Learning twice is different from learning once and from learning more. Neuroscience 132: 273-279.

Cohen-Matsliah, S.I., Brosh, I., Rosenblum, K., and Barkai, E. 2007. A novel role for extracellular signal-regulated kinase in maintaining long-term memory-relevant excitability changes. J. Neurosci. 27: 12584-12589.

Colombo, P.J., Brightwell, J.J., and Countryman, R.A. 2003. Cognitive strategy-specific increases in phosphorylated cAMP response element-binding protein and c-Fos in the hippocampus and dorsal striatum. J. Neurosci. 23: 3547-3554.

Countryman, R.A., Orlowski, J.D., Brightwell, J.J., Oskowitz, A.Z., and Colombo, P.J. 2005. CREB phosphorylation and c-Fos expression in the hippocampus of rats during acquisition and recall of a socially transmitted food preference. Hippocampus 15: 56-67.

Davis, H.P. and Squire, L.R. 1984. Protein synthesis and memory: A review. Psychol. Bull. 96: 518-559.

Desmedt, A., Hazvi, S., and Dudai, Y. 2003. Differential pattern of cAMP response element-binding protein activation in the rat brain after conditioned aversion as a function of the associative process engaged: Taste versus context association. J. Neurosci. 23: 6102-6110.

Dudai, Y. 1996. Consolidation: Fragility on the road to the engram. Neuron 17: $367-370$

Florian, C., Mons, N., and Roullet, P. 2006. CREB antisense oligodeoxynucleotide administration into the dorsal hippocampal CA3 region impairs long- but not short-term spatial memory in mice. Learn. Mem. 13: 465-472.

Franklin, B.J. and Paxinos, G. 1997. The mouse brain in stereotaxic coordinates. Academic Press, San Diego, CA.

Gill, K.M., Bernstein, I.L., and Mizumori, S.J. 2007. Immediate early gene activation in hippocampus and dorsal striatum: Effects of explicit place and response training. Neurobiol. Learn. Mem. 87: 583-596.

Goldbart, A., Row, B.W., Kheirandish, L., Schurr, A., Gozal, E., Guo, S.Z., Payne, R.S., Cheng, Z., Brittian, K.R., and Gozal, D. 2003. Intermittent hypoxic exposure during light phase induces changes in cAMP response element binding protein activity in the rat CA1 hippocampal region: Water maze performance correlates. Neuroscience 122: 585-590.

Grunbaum, L. and Muller, U. 1998. Induction of a specific olfactory memory leads to a long-lasting activation of protein kinase $\mathrm{C}$ in the antennal lobe of the honeybee. J. Neurosci. 18: 4384-4392.

Guzowski, J.F. and McGaugh, J.L. 1997. Antisense oligodeoxynucleotide-mediated disruption of hippocampal cAMP response element binding protein levels impairs consolidation of memory for water maze training. Proc. Natl. Acad. Sci. 94: 2693-2698.

Guzowski, J.F., Setlow, B., Wagner, E.K., and McGaugh, J.L. 2001 Experience-dependent gene expression in the rat hippocampus after spatial learning: A comparison of the immediate-early genes Arc, c-fos, and zif268. J. Neurosci. 21: 5089-5098.

Guzowski, J.F., Miyashita, T., Chawla, M.K., Sanderson, J., Maes, L.I., Houston, F.P., Lipa, P., McNaughton, B.L., Worley, P.F., and Barnes, C.A. 2006. Recent behavioral history modifies coupling between cell activity and $\operatorname{Arc}$ gene transcription in hippocampal CA1 neurons. Proc. Natl. Acad. Sci. 103: 1077-1082.

Impey, S., Smith, D.M., Obrietan, K., Donahue, R., Wade, C., and Storm, D.R. 1998. Stimulation of cAMP response element (CRE)-mediated transcription during contextual learning. Nat. Neurosci. 1: 595-601.

Izquierdo, I. and Medina, J.H. 1997. Memory formation: The sequence of biochemical events in the hippocampus and its connection to activity in other brain structures. Neurobiol. Learn. Mem. 68: $285-316$

Izquierdo, L.A., Viola, H., Barros, D.M., Alonso, M., Vianna, M.R., Furman, M., Levi de Stein, M., Szapiro, G., Rodrigues, C. , Choi, H., et al. 2001. Novelty enhances retrieval: Molecular mechanisms involved in rat hippocampus. Eur. J. Neurosci. 13: 1464-1467.

Kesner, R.P., Lee, I., and Gilbert, P. 2004. A behavioral assessment of hippocampal function based on a subregional analysis. Rev. Neurosci. 15: $333-351$

Kida, S., Josselyn, S.A., de Ortiz, S.P., Kogan, J.H., Chevere, I., Masushige, S., and Silva, A.J. 2002. CREB required for the stability of new and reactivated fear memories. Nat. Neurosci. 5: 348-355.

Kogan, I. and Richter-Levin, G. 2008. Activation pattern of the limbic system following spatial learning under stress. Eur. J. Neurosci. 27: 715-722.
Malleret, G., Hen, R., Guillou, J.L., Segu, L., and Buhot, M.C. 1999. 5-HT1B receptor knock-out mice exhibit increased exploratory activity and enhanced spatial memory performance in the Morris water maze. J. Neurosci. 19: 6157-6168.

Martel, G., Millard, A., Jaffard, R., and Guillou, J.L. 2006. Stimulation of hippocampal adenylyl cyclase activity dissociates memory consolidation processes for response and place learning. Learn. Mem. 13: $342-348$.

Martel, G., Blanchard, J., Mons, N., Gastambide, F., Micheau, J., and Guillou, J.L. 2007. Dynamic interplays between memory systems depend on practice: The hippocampus is not always the first to provide solution. Neuroscience 150: 743-753.

Matthies, H. 1989. In search of cellular mechanisms of memory. Prog. Neurobiol. 32: 277-349.

McGaugh, J.L. 1966. Time-dependent processes in memory storage. Science 153: 1351-1358.

McGaugh, J.L. 2000. Memory-a century of consolidation. Science 287: 248-251.

Miyashita, T., Kubik, S., Lewandowski, G., and Guzowski, J.F. 2008. Networks of neurons, networks of genes: An integrated view of memory consolidation. Neurobiol. Learn. Mem. 89: 269-284.

Mizuno, M., Yamada, K., Maekawa, N., Saito, K., Seishima, M., and Nabeshima, T. 2002. CREB phosphorylation as a molecular marker of memory processing in the hippocampus for spatial learning. Behav. Brain Res. 133: 135-141.

Moncada, D. and Viola, H. 2006. Phosphorylation state of CREB in the rat hippocampus: A molecular switch between spatial novelty and spatial familiarity? Neurobiol. Learn. Mem. 86: 9-18

Morris, R. 1984. Developments of a water-maze procedure for studying spatial learning in the rat. J. Neurosci. Methods 11: 47-60.

Morris, R.G., Garrud, P., Rawlins, J.N., and O'Keefe, J. 1982. Place navigation impaired in rats with hippocampal lesions. Nature 297: 681-683.

Muller, U. 2000. Prolonged activation of cAMP-dependent protein kinase during conditioning induces long-term memory in honeybees. Neuron 27: 159-168.

Packard, M.G. and McGaugh, J.L. 1992. Double dissociation of fornix and caudate nucleus lesions on acquisition of two water maze tasks: Further evidence for multiple memory systems. Behav. Neurosci. 106: $439-446$.

Packard, M.G. and Teather, L.A. 1997. Double dissociation of hippocampal and dorsal-striatal memory systems by posttraining intracerebral injections of 2-amino-5-phosphonopentanoic acid. Behav. Neurosci. 111: 543-551.

Packard, M.G., Hirsh, R., and White, N.M. 1989. Differential effects of fornix and caudate nucleus lesions on two radial maze tasks: Evidence for multiple memory systems. J. Neurosci. 9: 1465-1472.

Packard, M.G., Cahill, L., and McGaugh, J.L. 1994. Amygdala modulation of hippocampal-dependent and caudate nucleus-dependent memory processes. Proc. Natl. Acad. Sci. 91: 8477-8481.

Pittenger, C., Huang, Y.Y., Paletzki, R.F., Bourtchuladze, R., Scanlin, H., Vronskaya, S., and Kandel, E.R. 2002. Reversible inhibition of CREB/ATF transcription factors in region CA1 of the dorsal hippocampus disrupts hippocampus-dependent spatial memory. Neuron 34: 447-462.

Poirier, G.L., Amin, E., and Aggleton, J.P. 2008. Qualitatively different hippocampal subfield engagement emerges with mastery of a spatial memory task by rats. J. Neurosci. 28: $1034-1045$.

Porte, Y., Buhot, M.C., and Mons, N. 2008. Alteration of CREB phosphorylation and spatial memory deficits in aged 129T2/Sv mice. Neurobiol. Aging 29: 1533-1546.

Ramirez-Amaya, V., Vazdarjanova, A., Mikhael, D., Rosi, S., Worley, P.F., and Barnes, C.A. 2005. Spatial exploration-induced Arc mRNA and protein expression: Evidence for selective, network-specific reactivation. J. Neurosci. 25: 1761-1768.

Remondes, M. and Schuman, E.M. 2004. Role for a cortical input to hippocampal area CA1 in the consolidation of a long-term memory. Nature 431: 699-703.

Roozendaal, B., Hahn, E.L., Nathan, S.V., de Quervain, D.J., and McGaugh, J.L. 2004. Glucocorticoid effects on memory retrieval require concurrent noradrenergic activity in the hippocampus and basolateral amygdala. J. Neurosci. 24: 8161-8169.

Schulz, S., Siemer, H., Krug, M., and Hollt, V. 1999. Direct evidence for biphasic cAMP responsive element-binding protein phosphorylation during long-term potentiation in the rat dentate gyrus in vivo. $J$. Neurosci. 19: 5683-5692.

Silva, A.J., Kogan, J.H., Frankland, P.W., and Kida, S. 1998. CREB and memory. Аnnu. Rev. Neurosci. 21: 127-148.

Stanciu, M., Radulovic, J., and Spiess, J. 2001. Phosphorylated cAMP response element binding protein in the mouse brain after fear conditioning: Relationship to Fos production. Brain Res. Mol. Brain 
Res. 94: $15-24$.

Sutherland, R.J., Weisend, M.P., Mumby, D., Astur, R.S., Hanlon, F.M., Koerner, A., Thomas, M.J., Wu, Y., Moses, S.N., Cole, C., et al. 2001. Retrograde amnesia after hippocampal damage: Recent vs. remote memories in two tasks. Hippocampus 11: 27-42.

Swank, M.W. and Sweatt, J.D. 2001. Increased histone acetyltransferase and lysine acetyltransferase activity and biphasic activation of the ERK/RSK cascade in insular cortex during novel taste learning. $J$. Neurosci. 21: 3383-3391.

Taubenfeld, S.M., Wiig, K.A., Bear, M.F., and Alberini, C.M. 1999. A molecular correlate of memory and amnesia in the hippocampus. Nat. Neurosci. 2: 309-310.

Taubenfeld, S.M., Wiig, K.A., Monti, B., Dolan, B., Pollonini, G., and Alberini, C.M. 2001. Fornix-dependent induction of hippocampal CCAAT enhancer-binding protein $\beta$ and $\delta$ co-localizes with phosphorylated cAMP response element-binding protein and accompanies long-term memory consolidation. J. Neurosci. 21: 84-91.

Teather, L.A., Packard, M.G., Smith, D.E., Ellis-Behnke, R.G., and Bazan, N.G. 2005. Differential induction of c-Jun and Fos-like proteins in rat hippocampus and dorsal striatum after training in two water maze tasks. Neurobiol. Learn. Mem. 84: 75-84.

Trifilieff, P., Herry, C., Vanhoutte, P., Caboche, J., Desmedt, A., Riedel, G., Mons, N., and Micheau, J. 2006. Foreground contextual fear memory consolidation requires two independent phases of hippocampal ERK/CREB activation. Learn. Mem. 13: 349-358.

Trifilieff, P., Calandreau, L., Herry, C., Mons, N., and Micheau, J. 2007. Biphasic ERK1/2 activation in both the hippocampus and amygdala may reveal a system consolidation of contextual fear memory. Neurobiol. Learn. Mem. 88: 424-434.

Vianna, M.R., Alonso, M., Viola, H., Quevedo, J., de Paris, F., Furman, M., de Stein, M.L., Medina, J.H., and Izquierdo, I. 2000. Role of hippocampal signaling pathways in long-term memory formation of a nonassociative learning task in the rat. Learn. Mem. 7: 333-340.

Zhang, J.J., Okutani, F., Inoue, S., and Kaba, H. 2003. Activation of the cyclic AMP response element-binding protein signaling pathway in the olfactory bulb is required for the acquisition of olfactory aversive learning in young rats. Neuroscience 117: 707-713.

Received June 6, 2008; accepted in revised form October 16, 2008. 


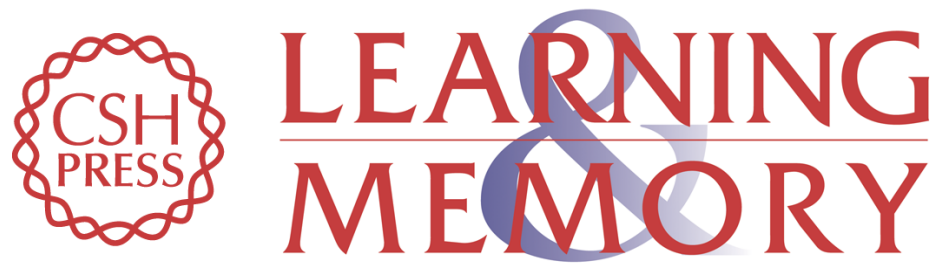

\section{Spatial memory in the Morris water maze and activation of cyclic AMP response element-binding (CREB) protein within the mouse hippocampus}

Yves Porte, Marie Christine Buhot and Nicole E. Mons

Learn. Mem. 2008, 15:

Access the most recent version at doi:10.1101/lm.1094208

References This article cites 67 articles, 28 of which can be accessed free at: http://learnmem.cshlp.org/content/15/12/885.full.html\#ref-list-1

License

Email Alerting

Receive free email alerts when new articles cite this article - sign up in the box at the Service top right corner of the article or click here. 